OPEN ACCESS

Edited by:

Marc Thibonnier,

AptamiR Therapeutics. Inc.,

United States

Reviewed by:

Soonkyu Chung,

University of Massachusetts Amherst,

United States

Naima Moustaid-Moussa,

Texas Tech University, United States

*Correspondence:

Jun Wada

junwada@okayama-u.ac.jp

Specialty section:

This article was submitted to

Obesity,

a section of the journal

Frontiers in Endocrinology

Received: 30 July 2021 Accepted: 07 December 2021 Published: 03 January 2022

Citation:

Yamaguchi S, Zhang D, Katayama $A$,

Kurooka N, Sugawara $R$,

Albuayjan HHH, Nakatsuka A, Eguchi J and Wada J (2022) Adipocyte-

Specific Inhibition of Mir221/222

Ameliorates Diet-Induced Obesity

Through Targeting Ddit4.

Front. Endocrinol. 12:750261.

doi: $10.3389 /$ fendo.2021.750261

\section{Adipocyte-Specific Inhibition of Mir221/222 Ameliorates Diet-Induced Obesity Through Targeting Ddit4}

\author{
Satoshi Yamaguchi, Dongxiao Zhang, Akihiro Katayama, Naoko Kurooka, \\ Ryosuke Sugawara, Haya Hamed Hassan Albuayjan, Atsuko Nakatsuka, \\ Jun Eguchi and Jun Wada*
}

Department of Nephrology, Rheumatology, Endocrinology and Metabolism, Okayama University Graduate School of Medicine, Dentistry and Pharmaceutical Sciences, Okayama, Japan

MicroRNAs expressed in adipocytes are involved in transcriptional regulation of target mRNAs in obesity, but miRNAs critically involved in this process is not well characterized. Here, we identified upregulation of miR-221-3p and miR-222-3p in the white adipose tissues in C57BL/6 mice fed with high fat-high sucrose (HFHS) chow by RNA sequencing. Mir221 and Mir222 are paralogous genes and share the common seed sequence and Mir221/222AdipoKO mice fed with HFHS chow demonstrated resistance to the development of obesity compared with Mir221/222 flox/y. Ddit4 is a direct target of Mir221 and Mir222, and the upregulation of Ddit4 in Mir221/222AdipoKO was associated with the suppression of TSC2 (tuberous sclerosis complex 2)/mammalian target of rapamycin complex 1 (mTORC1)/S6K (ribosomal protein S6 kinase) pathway. The overexpression of miR-222-3p linked to enhanced adipogenesis, and it may be a potential candidate for miRNA-based therapy.

Keywords: non-coding RNAs, microRNA, adipose tissues, Adipogenesis, mTORC1

\section{INTRODUCTION}

In obesity, the excess and ectopic accumulation of adipose tissue leads to the clusters of metabolic disorders such as low-grade inflammation, type 2 diabetes (T2D), dyslipidemia, hypertension, nonalcoholic fatty liver disorder (NAFLD), cardiovascular diseases (CVDs), chronic kidney disease (CKD) and cancer. As an endocrine organ, the adipose tissue communicates with other tissues by secreting peptide hormones, inflammatory cytokines, signaling lipids and miRNAs packed in exosomes (1). The alteration of expression profile of miRNA both in cytoplasm and extracellular vesicles leads to changes in the transcriptional and translational activities of the genes, which control inflammation, whole body insulin sensitivity, lipid metabolism, adipogenesis of white, beige, and brown adipose tissues (2). For instance, miR-34a secreted by mature adipocytes in exosomes were transported into macrophages, suppresses M2 polarization, and stimulates inflammatory responses by repressing Krüppel-like factor 4 (3). Lentivirus-mediated suppression of miR-34a increased the beige and brown fat formation in diet-induced obese mice by increasing fibroblast growth factor 21 and sirtuin 1 (4). The genetic ablation of miR-128-1 in mouse metabolic disease models resulted in increased energy expenditure, amelioration of diet-induced obesity and enhanced insulin sensitivity (5). In lipid metabolism, miR-425 overexpression in mice resulted in the promotion of diet-induced 
obesity and overexpression of miR-425 in 3T3-L1 cells accelerated adipogenesis and lipogenesis, while knock down of miR-425 remarkably enhanced lipolysis and lipid oxidation (6).

We also attempted to identify specific miRNAs critically involved in the process of obesity, we surveyed expression profile of miRNAs in liver, muscle, white adipose tissues (WATs), and sera of C57BL/6JJcl mice fed with standard (STD) and high fat-high sucrose (HFHS) chow by RNA sequencing (GSE61959) (7). miR-221-3p and miR-222-3p are highly up-regulated in epididymal adipose tissues in mice fed with high fat high sucrose (HFHS). miR-221 and miR-222 both locate in the close proximity on the $\mathrm{X}$ chromosome and share the identical seed sequence (8). In meta-analysis, circulating miR221-3p was reduced in T2D, while miR-222-3p was elevated in obesity and T2D (9). Although miR-221-3p and miR-222-3p are differentially expressed in sera and adipose tissues, the functional in vivo experiments to elucidate the roles of Mir221 and Mir222 in obesity has not been reported. Here, we investigated the adipocyte specific Mir221 and Mir222 knockout (Mir221/ 222AdipoKO) mice fed with HFHS chow, and they were protected from the development of obesity by targeting DDIT4 (DNA damage inducible transcript 4)/TSC2 (tuberous sclerosis complex 2)/S6K (ribosomal protein S6 kinase) pathway.

\section{MATERIALS AND METHODS}

\section{Animal Models}

We obtained Mir $221 \mathrm{KO}$ cond (Mir221 ${ }^{\mathrm{tm} 2}$; EMMA ID, EM:05507) from EMMA (the European Mouse Mutant Archive) and Helmholz Zentrum München (Neuherberg, Germany). The targeting vector cassette composed of Neo flanked by FRT and distal loxP sites was inserted outside the genomic region encoding Mir221 and Mir222 precursors. Neo was removed by Flp in the conditional KO allele of Mir $221 \mathrm{KO}$ cond. The conditional KO allele was confirmed by primer pair; 1544_29 (5'- GCT CTG TTT TCC TAA GTG ATG G-3') and 1544_30 (5'-CTG ACA GGA AGT AAA TCA TCT TAG C-3'). The expected fragments were $265 \mathrm{bp}$ in wild and $384 \mathrm{bp}$ in conditional KO allele. We also used B6.FVB-Tg (Adipoq-cre) 1 Evdr/J to produce Mir221/222AdipoKO mice by crossing to Mir $221 \mathrm{KO}$ cond. Adipoq-cre transgene was screened by primers, Forward (5'-AGC GAT GGA TTT CCG TCT CT-3') and Reverse (5'-CAC CAG CTT GCA TGA TCT CC-3'). The primers, oIMR7338 (5'-CTA GGC CAC AGA ATT GAA AGA TCT-3') and oIMR7339 (5'-GTA GGT GGA AAT TCT AGC ATC ATC C-3'), were used for internal positive control. The expected sizes for transgene and internal positive control were $200 \mathrm{bp}$ and 324 $\mathrm{bp}$, respectively. By crossing male Tg (Adipoq-cre) and female Mir221 $1^{\mathrm{tm} 2} / y$ C57BL/6JJcl mice, we generated male Mir221/ $222^{\text {flox/y }}$ and male Mir221/222AdipoKO littermates.

Five-week-old mice were randomly assigned to standard diet (STD) group (MF, Oriental Yeast, Japan) or high fat high sucrose diet (HFHS) group (D12331, Research Diets, New Brunswick, NJ). The detailed formulation of STD (10) (https://www.oyc.co.jp/bio/LAD-equipment/ LAD/ingredient.html and https://www.oyc.co.jp/bio/LAD-
equipment/LAD/rodents.html) and HFHS (https://www.eptrading. co.jp/service/researchdiets/pdf/D12328-D12331.pdf) diets are shown in Supplementary Table 1. At 22 weeks of age, we obtained various organs and they were subjected to following experiments.

\section{T3-L1 Cell Cultures}

3T3-L1 pre-adipocytes were cultured in Dulbecco's modified eagle's medium (DMEM, 2124951, Gibco). On day 0, the cells were treated with the differentiation media; DMEM supplemented with $10 \% \mathrm{FBS}, 10 \mu \mathrm{g} / \mathrm{ml}$ insulin (I1882, Sigma), $1 \mu$ M DEX (D2915, Sigma) and 0.5 mM IBMX (I5879, Sigma). Then, the media were changed to DMEM supplemented with $10 \mu \mathrm{g} / \mathrm{ml}$ insulin and $10 \%$ FBS on day 2 and cultured for 10 or 20 days. Undifferentiated 3T3-L1 cells were subjected to Lentiviral miRNA expression, Lentiviral miRNA inhibition studies, and luciferase reporter assays.

\section{Human Serum Samples}

Human serum samples were collected from 69 patients with type 2 diabetes in Okayama University Hospital and 45 subjects with normal fasting glucose (NGT).

\section{Insulin Tolerance Test and Glucose Tolerance Test (ITT and GTT)}

The 13-week-old mice were fasted for 16 hours in GTT and for 3 hours in ITT. They were then intraperitoneally injected with glucose solution ( $1 \mathrm{mg} / \mathrm{g}$ body weight) and human insulin (1 unit/kg in HFHS groups and 0.75 unit/kg in STD groups) for GTT and ITT, respectively.

\section{Basal Metabolic Rate, Locomotor Activity, and Food Intake}

At 18 weeks of age, $\mathrm{O}_{2} / \mathrm{CO}_{2}$ metabolism measuring system (MK5000, Muromachi Kikai, Tokyo, Japan) were used to quantify oxygen consumption rate and carbon dioxide production for the estimation of $\dot{\mathrm{VO}} 2$ and respiratory quotient (RQ). The locomotor activity was recorded for 24 hours by the frequency of interrupting an infrared sensor (ACTIMO-100, Shinfactory, Fukuoka, Japan). Daily food intake was measured and calculated; daily food intake [g/ day/body weight $(\mathrm{BW})]=$ [initial food weight $(\mathrm{g})$ leftover food weight (g)]/measurement period (days)/BW (g). The 6-10 mice in each experimental group were examined.

\section{Reverse Transcription-Quantitative Polymerase Chain Reaction (RT-qPCR)}

RNAs were extracted from frozen tissues and cultured 3T3-L1 cells with RNeasy Mini kit (74106, Qiagen). The QIAamp Circulating Nucleic Acid Kit (Qiagen) were used for the isolation of total RNAs from serum. For gene expression analyses, cDNAs were prepared with High-Capacity RNA-to-cDNA Kit (Thermo Fisher Scientific). TaqMan gene expression primers, Ddit4 (Mm00512504_g1), Rplp0 (Mm00725448_s1), Rn18s (Mm03928990_g1), Adipoq (Mm00456425_m1), Lep (Mm00434759_m1), Lpl (Mm01345523_m1), Srebf1 (Mm00550338_m1), Cebpa (Mm00514283_s1), Fabp4 (Mm00445878_m1), Pparg (Mm01184322_m1), Lipe (Mm00495359_m1), Pnpla2 
(Mm00503040_m1), Ucp1 (Mm01244861_m1), Cox8b (Mm00432648_m1), Prdm16 (Mm00712556_m1), Cidea (Mm00432554_m1), Ppargc1a (Mm01208835_m1), G6pc (Mm00839363_m1), Gck (Mm00439129_m1), Fasn (Mm00662319_m1), Ppara (Mm00440939_m1), Il6 (Mm00446190_m1), Ifng (Mm01168134_m1), Tnf (Mm00443258_m1), Il1b (Mm01336189_m1) were used (Thermo Fisher Scientific). For miRNA expression studies, cDNAs were prepared from total RNAs by TaqMan MicroRNA Reverse Transcription Kit (Life Technologies). MicroRNA primers, $m m u$ miR-222-3p (CTAAJ3), hsa-miR-221-3p (000524), hsa-miR-222-3p (002276), snoRNA202 (001232), snoRNA234 (001234), and celmiR-39 (000200) were used (Thermo Fisher Scientific). Rplp0, Rn18s, snoRNA202, snoRNA234, and cel-miR-39 were served as the invariant controls. The RT-qPCR was performed using TaqMan Universal PCR Master mix II (no UNG) at a StepOne Plus Real-Time PCR system. The quantification was performed by the $2^{-\Delta \Delta C T}$ analysis method.

\section{Cloning of Mir221 Host Gene (Mir221 hg)}

Long non-coding RNA, Mir221hg, was cloned using SMARTer RACE 5'/3' Kit (634858, Clontech). 5'- and 3'-RACE-Ready cDNAs from epididymal adipose tissues poly $\mathrm{A}^{+}$RNA were prepared by SMARTScribe Reverse Transcriptase. 5'- and 3'RACE-Ready cDNAs were amplified by PCR using 5' GSP (5'GAT TAC GCC AAG CTT CCA GCA GAC AAT GTA GC TGT TGC-3') and 3' GSP (5'-GAT TAC GCC AAG CTT TCC AGG TCT GGG GCA TGA ACC TG-3'), respectively. Furthermore, nested PCR was performed using 5' NGSP (5'-GAT TAC GCC AAG CTT GTA TGC CAG GTT CAT GCC CCA GAC-3') and 3' NGSP (5'-GAT TAC GCC AAG CTT GCA ACA GCT ACA TTG TCT GCT GG-3'). PCR products were analyzed by agarose/ EtBr gel and purified by NucleoSpin Gel and PCR Clean-Up Kit. The purified RACE products were subcloned into $\mathrm{pRACE}$ vector by In-Fusion Cloning (Clontech). Independent 4 clones of both 5'- and 3'-RACE were sequenced.

\section{Western Blot Analysis}

The epididymal fat tissues from 22-week-old mice and cultured 3T3-L1 cells were homogenized in RIPA lysis buffer (radioimmunoprecipitation buffer) plus protease inhibitors. The samples were boiled in SDS-PAGE loading buffer, separated on 12\% Mini-PROTEAN TGX Precast Protein Gels (Bio-Rad), and transferred to a PVDF Blotting Membrane (cytiva). After blocking with $5 \%$ nonfat milk for 1 hour at room temperature (RT), the blots were incubated with REDD-1/DDIT4 Antibody, rabbit polyclonal (ab106356, RRID: AB_10864294), C/EBP $\alpha$ Antibody, rabbit polyclonal (2295, RRID: AB_10692506), PPAR $\gamma$ (D69) Antibody, rabbit polyclonal (2430, RRID: AB_823599), mTOR (7C10) Rabbit mAb (2983, RRID: AB_2105622), Phospho-mTOR (Ser2448) (D9C2) XP Rabbit mAb (5536, RRID: AB_10691552), Akt Antibody, rabbit polyclonal (9272, RRID: AB_329827), PhosphoAkt (Thr308) Antibody, rabbit polyclonal (9275, RRID: AB_329828), p70 S6 Kinase (49D7) Rabbit mAb (2708, RRID: AB_390722), Phospho-p70 S6 Kinase (Thr389) Antibody, rabbit polyclonal (9205, RRID: AB_330944), Tuberin/TSC2 Antibody, rabbit polyclonal (3612, RRID: AB_2207804), Phospho-Tuberin/
TSC2 (Thr1462) Antibody, rabbit polyclonal (3611, RRID: AB_329855, Cell Signaling Technology) overnight at $4^{\circ} \mathrm{C}$. GAPDH (D16H11) XP Rabbit mAb (HRP Conjugate) (8884, RRID: AB_11129865) was used as a loading control (Cell Signaling Technology). After washing three times with Trisbuffered saline (TBS), the blots were incubated with ECL Donkey Anti-Rabbit IgG, HRP-Conjugated Antibodies (NA934V, GE healthcare Life science, 1:10000) at RT for 1 hour. The blots were developed with Pierce ECL Western Blotting Substrate (TE261327, Thermo Fisher Scientific). The chemiluminescence was analyzed using ImageQuant LAS-4000 mini (FUJIFILM).

\section{Morphometric Analysis for Adipocyte Size}

Epidydimal adipose tissues were fixed by $10 \%$ formalin, embedded with paraffin. The $5-\mu \mathrm{m}$ paraffin sections were prepared and stained with PAS. The images were captured using an Olympus BX51 microscope. The size of the adipocytes was analyzed by Keyence Hybrid cell count software. Epidydimal adipose tissues were taken from 3-5 individual animals from each experimental group.

\section{Isolation of Stromal Vascular Fraction (SVF) From White Adipose Tissues}

SVF was isolated from epididymal adipose tissue of 24-week-old mice. Briefly, fresh mouse epididymal fat pads were minced and digested with collagenase type 1 (CLSS1, Worthington) in HBSS containing $10 \%$ FBS for 45 minutes at $37^{\circ} \mathrm{C}$. The mixture was filtered through a nylon mesh $(100 \mu \mathrm{m})$, then centrifuged at $400 \mathrm{~g}$ for 1 minute. The adipocyte fraction was obtained from the supernatant, they were again centrifuged at $800 \mathrm{~g}$ for 10 minutes, and the SVF was obtained from the pellet.

\section{Identification of Mir221/222 Target mRNAs}

The mRNA microarray was performed by GeneChip Mouse Gene 2.0 array using total RNA of epidydimal fat obtained from 16-week-old mice (1 animal from each group) and analyzed by Filgen (Nagoya, Japan). The raw data are available in Gene Expression Omnibus (GEO; https://www.ncbi.nlm.nih.gov/geo/) (GSE163921). TargetScan (http://www.targetscan.org/vert_72/), miRDB (http://www.mirdb.org/), and DIANA-microT v5.0 (https://bio.tools/DIANA-microT) and were used to identify potential target genes for Mir221/222.

\section{Lentiviral miRNA Expression and Lentiviral miRNA Inhibitor}

Lentiviral transduction using pLV-miRNA and pLV-miR-Locker system to 3T3-L1 cells were performed according to the manufacturer's manual (Biosettia). After transformation to $E$. coli JM109 cells, pLV-[mmu-mir-221] plasmid (mir-p177m, Biosettia), pLV-[mmu-mir-222] plasmid (mir-p178m, Biosettia), pLV-[mmu-mir-221-3p] locker plasmid (mirmp0337, Biosettia), pLV-[mmu-mir-222-3p] locker plasmid (mir-mp0339, Biosettia), pLV-[mir-control] plasmid (mirp000, Biosettia), pLV-miR-locker control plasmid (mir-lockerctrl, Biosettia), pMDLg/pRRE (12251, Addgene), pRSV/Rev (12253, Addgene), and pMD2.G (12259, Addgene) were isolated with EndoFree Plasmid Maxi Kit (12362, Qiagen). 
$293 \mathrm{~T}$ cells $\left(10 \times 10^{7} / 5 \mathrm{~mL}\right)$ were transfected with each $\mathrm{pLV}$ plasmid, pMDLg/pRRE, pRSV/Rev, pMD2.G, Lipofectamine LTX, and $1.5 \mathrm{~mL}$ Opti-MEM. The supernatants were collected after 48 hours after transfection. 3T3-L1 cells were transduced with lentivirus stock in complete media containing $10 \mu \mathrm{g} / \mathrm{mL}$ polybrene for 12 hours, replaced with fresh complete medium

\section{Luciferase Reporter Assay}

To quantitatively evaluate miRNA activity on cloned miRNA target sequence from 3'-untranslated region (3'-UTR) of Ddit4, pmirGLO dual luciferase miRNA Target expression vector (E1330, Promega) was used. Firstly, the pmirGLO plasmid was linearized by double digestion with XhoI and SalI. The cDNA of Ddit4 wild type (WT) 3'-UTR was amplified by PCR and ligated with CIP treated pmirGLO Vector. The primers are Forward-XhoI-3'UTR-Ddit4: 5'-GGG GGG CTC GAG CAG CTG CTC ATT GAA GAG TG-3', and Reverse-SalI-3'UTR-Ddit4: 5'-GGG GGG GTCGAC CAA ACC AAC AGA GGA GAC AG-3'. pmirGLO-Ddit4 MT 3'-UTR was prepared by site directed mutagenesis by PCR using primers; Forward-MT-Seed-Ddit4: 5'-CTG GAT GTG TAT CTG CAT GTA C-3' and Reverse-MT-Seed-Ddit4: 5'-GTA CAT GCA GAT ACA CAT CCA G-3'. The seed sequence "CGATGTA" was mutated to "CGTCTA". After transformation to E. coli JM109 cells, pmirGLODdit4 WT 3'-UTR, pmirGLO-Ddit4 MT 3'-UTR, and pmirGLO no-insert control plasmids were isolated with EndoFree Plasmid Maxi Kit (12362, Qiagen). 3T3-L1 cells were seeded at a density of 120,000 cells/mL, then co-transfected with Syn-mmu-miR-221-3p (MIMAT0000669, Qiagen), Syn-mmu-miR-222-3p (MIMAT0000670, Qiagen), negative control siRNA (1027280, Qiagen), inhibitor negative control (1027271, Qiagen), pmirGLODdit4 WT 3'-UTR, pmirGLO-Ddit4 MT 3'-UTR, and pmirGLO no-insert control plasmids. Twenty-four hours after transfection, the cells were analyzed to measure luciferase activities using the Dual-Glo Luciferase Assay System and a GloMax 20/20 luminometer (Promega).

\section{Statistical Analysis}

All values were represented as the mean \pm standard deviation (SD). Statistical analyses were conducted using IBM SPSS Statistics 23 and GraphPad Prism (version 8.0). Independent $t$ test, Mann-Whitney's U test, and one-way ANOVA with Tukey test was used to determine the differences. For correlation, nonparametric Spearman $\mathrm{r}$ coefficient was used. $\mathrm{p}<0.05$ was considered statistically significant.

\section{RESULTS}

\section{Mir221/222AdipoKO Mice Are Resistant to Diet-Induced Obesity}

To identify miRNAs critically involved in the disease process of obesity and type 2 diabetes (T2D), we performed miRNA profiling of serum, liver and epididymal fat tissues in C57BL/6JJcl mice fed with standard (STD) and high fat-high sucrose (HFHS) chow. The Illumina RNA sequencing data (Gene Expression Omnibus number GSE61959) demonstrated that the read numbers of Mir221 and
Mir222 were 5.7 and 8.2-fold up-regulated in epididymal adipose tissues in HFHS group compared with STD group (Supplementary Table 2). Mir221 and Mir222 are paralog genes located in proximity on $\mathrm{X}$ chromosome and they share identical seed sequence. To further investigate the role of Mir221/222 in obesity and diabetes, we crossed male Tg (Adipoq-cre) mice and female Mir221 ${ }^{\text {tm2/y }}$ C57BL/ $6 \mathrm{JJCl}$ mice and generated male Mir221/222 flox/y and male Mir221/ 222AdipoKO littermates.

Body weight of Mir221/222AdipoKO mice fed with HFHS chow was significantly reduced compared with Mir221/222 flox/y mice (Figure 1A). The weight of epididymal, mesenteric, subdermal, and brown fat was also reduced in Mir221/222AdipoKO mice (Figure 1B). The Mir221/222 $2^{\text {flox/y }}$ and Mir221/222AdipoKO mice fed with STD chow demonstrated no significant differences in their body and tissue weight (Figures 1A, B). The average size of adipocytes in epididymal adipose tissues derived from Mir221/ 222AdipoKO mice fed with HFHS chow was smaller compared with Mir221/222 $2^{\text {flox/y }}$ mice (Figure 1C). To investigate glucose homeostasis, we performed insulin tolerance test (ITT) and glucose tolerance test (GTT). The blood glucose levels of Mir221/ 222AdipoKO mice fed with HFHS chow were reduced in GTT and ITT compared with Mir221/222 flox/y, but they did not reach significant differences (Figure 1D). The serum insulin concentrations of Mir221/222AdipoKO mice were significantly lower than Mir221/222 flox/y , suggesting the improvement of insulin sensitivity in Mir221/222AdipoKO mice (Figure 1D). To investigate whether reduced adiposity in Mir221/222AdipoKO mice was due to changes in energy expenditure or energy intake, we measured basal metabolic rates, locomotor activity and food intake. Basal metabolic rate such as respiratory quotient and oxygen consumption were not altered between Mir221/222 flox/y and Mir221/222AdipoKO mice fed with HFHS chow (Supplementary Figures 1A, B). The locomotor activity was recorded for 24 hours, most of the activities were observed during the dark phase. Increased activity was observed in Mir221/222AdipoKO mice under HFHS chow compared with Mir221/222 $2^{\text {llox/y }}$ during light $(0.944 \pm 0.309$ vs $1.60 \pm 0.764$ counts/min, $\mathrm{p}=0.036)$ and dark (6.67 \pm 4.38 vs $7.94 \pm 4.38$ counts/min, $\mathrm{p}=0.497$ ) periods (Supplementary Figure 1C). Food consumptions were not altered in Mir221/ 222 AdipoKO and Mir221/222 flox/y fed with HFHS (Supplementary Figure 1D). Under HFHS chow, serum leptin concentrations were significantly reduced in Mir221/222AdipoKO compared with Mir221/222flox/y $(24.5 \pm 2.35$ vs $21.5 \pm 1.85 \mathrm{ng} / \mathrm{ml}$, $\mathrm{p}=0.043$ ), while serum adiponectin concentrations were not altered (Supplementary Figures 1E, F). In quantitative RT-PCR in epididymal adipose tissues, mRNA expression of Lep was significantly reduced in Mir221/222AdipoKO. The genes related to adipogenesis, such as Pparg, Cebpa, Ppargc1 and Prdm16, were not altered in Mir221/222AdipoKO (Supplementary Figure 2).

\section{Expression Mir221 and Mir222 Are Highly Induced by HFHS Chow Feeding in Mature Adipocytes From Epidydimal Adipose Tissue}

We investigated the expression of Mir221 and Mir222 in various organs. Both miR-221-3p and miR-222-3p were abundantly 
A
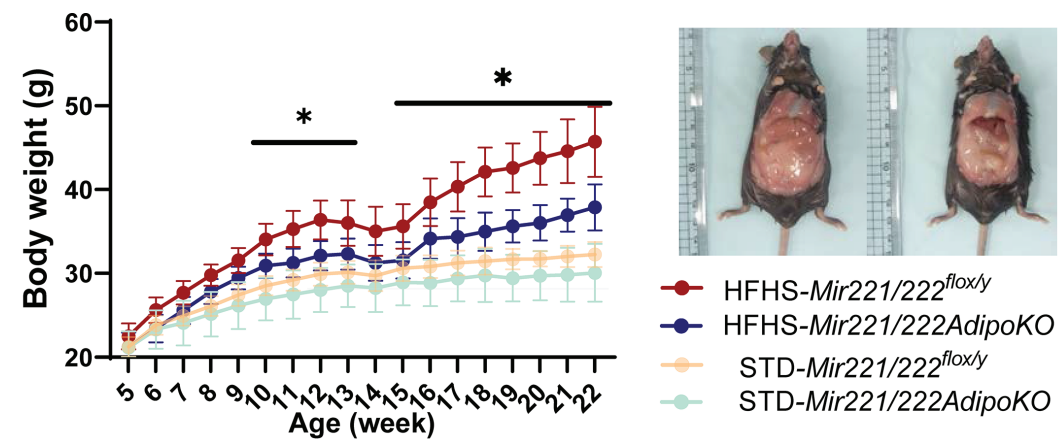

$\rightarrow$ HFHS-Mir221/222 flox/y

$\rightarrow$ HFHS-Mir221/222AdipoKO

STD-Mir221/222 flox/y

STD-Mir221/222AdipoKO

B

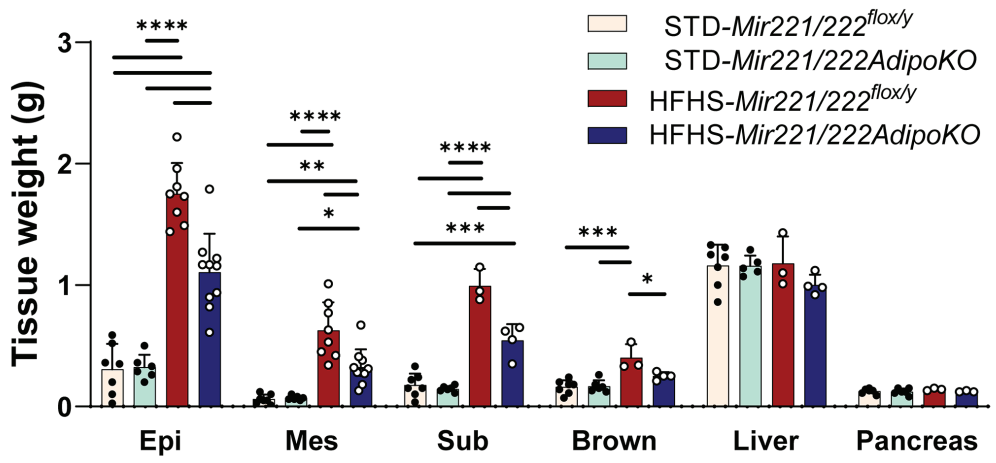

C

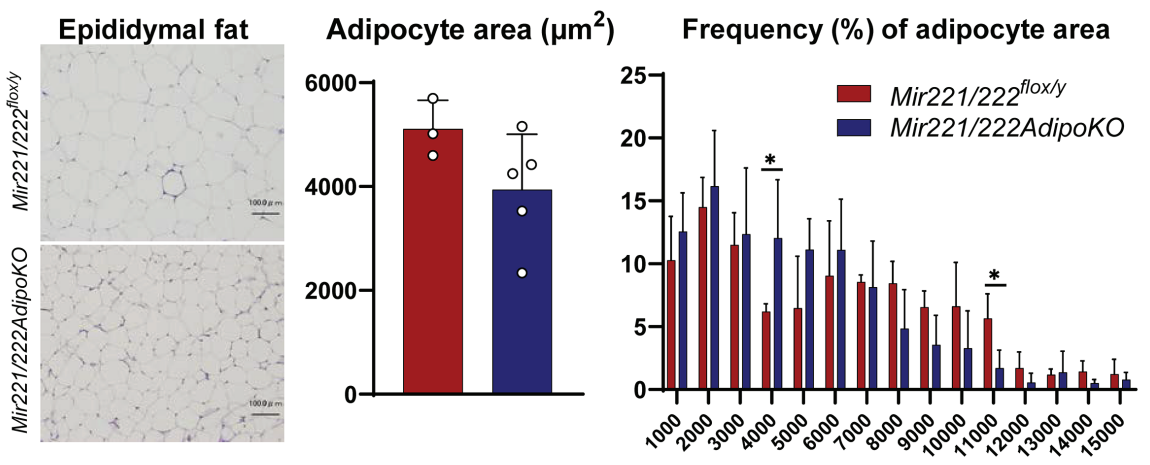

D

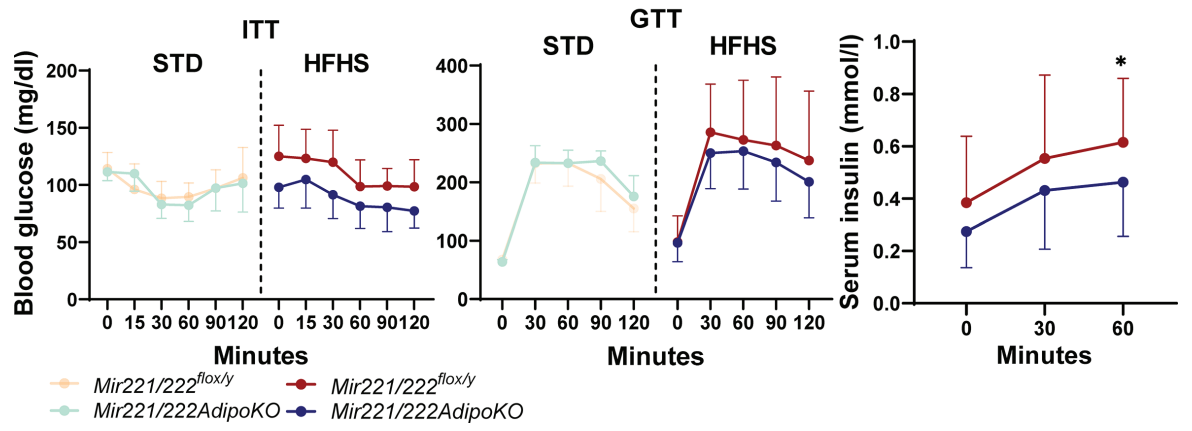

FIGURE 1 | The metabolic phenotypes of Mir221/222 flox/y and Mir221/222AdipoKO male mice fed with high fat high sucrose (HFHS) or standard (STD) chow. (A) Body weight of Mir221/222 floxy and Mir221/222AdipoKO mice fed with HFHS ( $n=7)$ or STD chow $(n=6)$, respectively. (B) Tissue weight of Mir221/222flox/y and Mir221/222AdipoKO mice fed with HFHS or STD chow at 24 weeks of age. (Epi, epididymal; Mes, mesenteric; Sub, inguinal; Brown, Brown adipose tissues) (C) Adipocyte area in epididymal adipose tissues of Mir221/222 fioxy $(n=3)$ and Mir221/222AdipoKO $(n=5)$ mice fed with HFHS or STD. Quantitative analyses were carried out on PAS-stained paraffin sections. (D) Insulin tolerance test (ITT) in Mir221/222flox/y ( $n=7)$ and Mir221/222AdipoKO ( $n=6)$. Glucose tolerance test (GTT) in Mir221/222flox/y fed with HFHS $(n=13)$ or STD chow $(n=7)$ and Mir221/222AdipoKO fed with HFHS $(n=16)$ or STD chow $(n=6)$. Data shown as mean \pm SD and analyzed by one-way ANOVA with Tukey test in (A, B), and Mann-Whitney's $U$ test in (C, D) ( $\left.{ }^{\star} p<0.05 ;{ }^{* *} \mathrm{p}<0.01 ;{ }^{* \star *} \mathrm{p}<0.001 ;{ }^{* \star *} \mathrm{p}<0.0001\right)$. 
expressed in brain, kidney, and lung in Mir221/222 flox/y and they were down-regulated or not altered by the HFHS feeding compared with STD chow. In contrast, both miR-221-3p and miR-222-3p were significantly upregulated in the epidydimal fat tissues, and such upregulation was canceled in the epidydimal fat tissues of Mir221/222AdipoKO fed with HFHS chow (Figures 2A, B). Next, we investigated the localization of Mir221 and Mir222 in the cell fractions of epididymal adipose tissues. Both miR-221-3p and miR-222-3p were significantly induced by HFHS chow in mature adipocytes of Mir221/ $222^{f l o x / y}$, while the upregulation was significantly reversed in the mature adipocyte of Mir221/222AdipoKO fed with HFHS chow. In contrast, miR-221-3p and miR-222-3p were not significantly upregulated in the stromal vascular fraction (SVF) from Mir221/222 flox/y fed with HFHS chow (Figure 2C). We also assessed the expression of miR-221-3p and miR-222-3p during 3T3-L1 adipocyte differentiation. Both miR-221-3p and miR222-3p continuously down-regulated after the induction of adipocyte differentiation (Supplementary Figure 3A). Finally, we assessed the serum concentrations of mmu-miR-221-3p and mmu-miR-222-3p by quantitative PCR and they were not altered in Mir221/222 $2^{\text {flox/y }}$ and Mir221/222AdipoKO fed with STD and

A

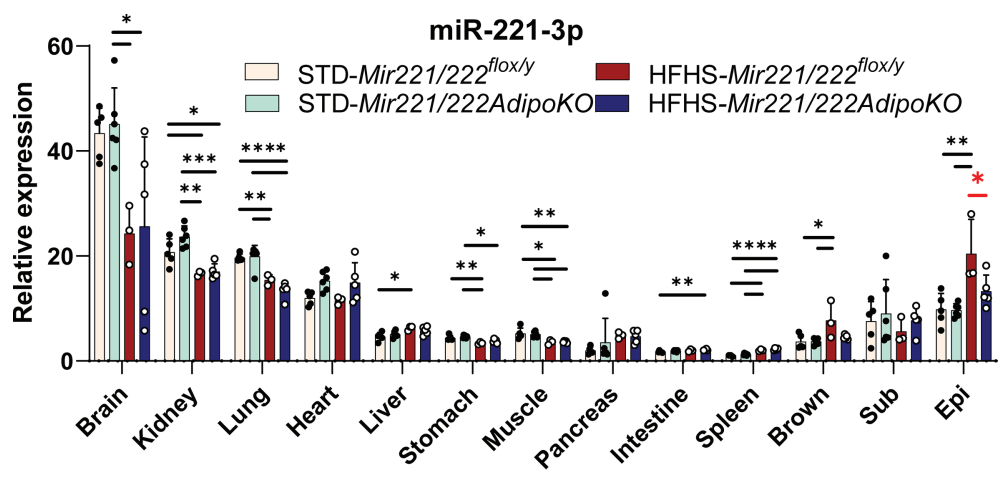

B

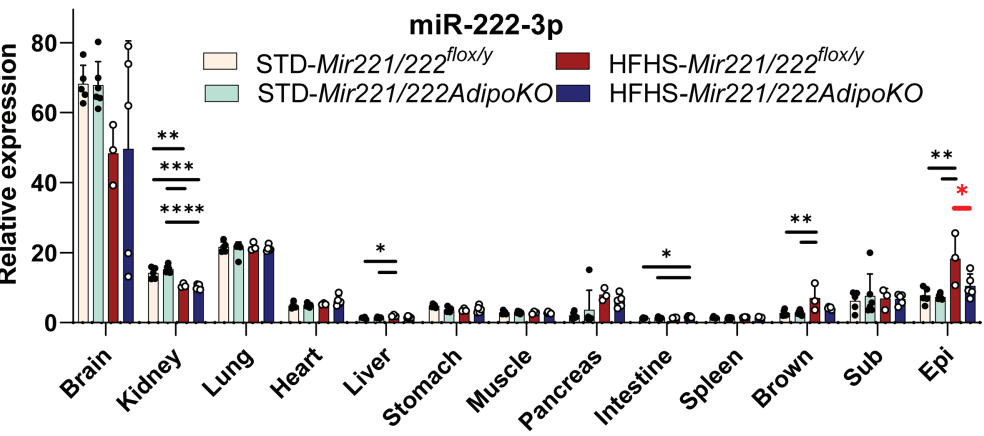

C

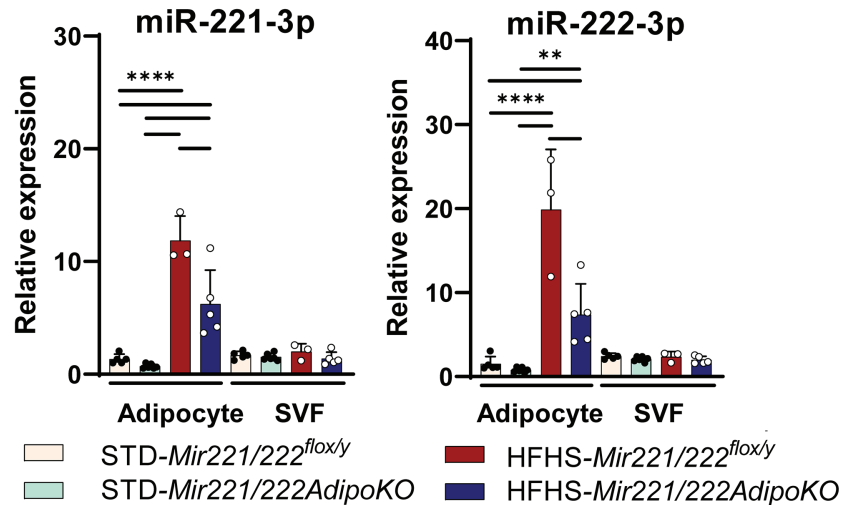

FIGURE 2 | Expression of Mir221 and Mir222 in Mir221/222 flox/y and Mir221/222AdipoKO male mice fed with high fat high sucrose (HFHS) or standard (STD) chow. (A, B) In various tissues, the expression of miR-221-3p and miR-222-3p is normalized by snoRNA202 and snoRNA234. The HFHS-induced up-regulation of miR221-3p and miR-222-3p in epididymal adipose tissues was reversed in Mir221/222AdipoKO (red asterisks). (Epi, epididymal; Mes, mesenteric; Sub, inguinal; Brown, Brown adipose tissues) (C) Serum concentration of miR-221-3p and miR-222-3p. HFHS Mir221/222 flox/y ( $n=3$ ), HFHS Mir221/222AdipoKO ( $n=5$ ), STD Mir221/ $222^{f l o x / y}(n=5)$ and STD Mir221/222AdipoKO mice $(n=6)$. Data shown as mean \pm SD and analyzed by one-way ANOVA with Tukey test $\left({ }^{*} p<0.05 ;{ }^{*} p<0.01\right.$; $\left.{ }^{* \star *} \mathrm{p}<0.001 ;{ }^{* \star \star} \mathrm{p}<0.0001\right)$. 
HFHS chow, suggesting serum mature forms of Mir221 and Mir222 were derived from various organs but not exclusively from adipose tissues (Supplementary Figure 4A). In human samples from the subjects with normal glucose tolerance (NGT, $\mathrm{n}=45$ ) and impaired glucose tolerance (IGT, $\mathrm{n}=69$ ), hsa-miR-221$3 p$ and hsa-miR-222-3p demonstrated negative $\left(R^{2}=0.1311\right.$, $\mathrm{p}<0.0001)$ and positive correlations $(\mathrm{R} 2=0.03841, \mathrm{p}=0.0441)$ with HbA1c levels, respectively (Supplementary Figures 4B, C).

\section{Identification of Mir221hg and Its Expression}

The miRNA genes are classified as intergenic and intragenic miRNAs, and the intragenic miRNAs and related host genes share the common transcriptional regulation. The long noncoding RNA (lncRNA), namely mir-221 host gene (MIR221HG), has been reported in bovine, which inhibits the adipocyte differentiation in cultured cells (11). Thus, we performed 5' and 3' rapid amplification of cDNA ends (5'/3'RACE) using poly $\mathrm{A}^{+}$RNA purified from epidydimal fat tissues of C57BL/6JJcl mice and cloned 1537 bp single exon lncRNA (Mir221hg, MW581002) which overlap Mir221 (Supplementary Figure 5A). Next, we designed primers for quantitative PCR of Mir221hg and evaluated gene expression in various tissue. Mir221hg was abundantly expressed in brain in Mir221/222 flox/y and they were upregulated in brain, heart, liver, intestine, spleen by the HFHS feeding compared with STD chow. The expression of Mir221hg was rather low in various adipose tissues and its lncRNA expression was not changed in Mir221/222AdipoKO fed with STD and HFHS chow (Supplementary Figures 5B, C). Although Mir221hg lncRNA may be functional as a reservoir for miR-221-3p, the role of Mir221 hg lncRNA in adipose tissues was limited in our experiments.

\section{miR-221-3p and miR-222-3p Target Ddit4}

To investigate the mechanism for the resistance to diet-induced obesity in Mir221/222AdipoKO, we attempted to identify the potential target genes for miR-221-3p and miR-222-3p. We performed mRNA profiling by GeneChip Mouse Gene 2.0 array using total RNA purified from epidydimal fat tissues of Mir221/222 flox/y fed with STD or HFHS, Mir221/222AdipoKO fed with STD or HFHS chow (Supplementary Tables 3-5). Then, we made the list of 355 target genes commonly predicted by TargetScan, miRDB, and DIANA-microT, and their seed sequences were conserved in both human and mouse genome (Supplementary Table 6). By searching upregulated genes in Mir221/222AdipoKO compared with Mir221/222 $2^{\text {lox/y }}$ in both STD and HFHS fed groups, we identified that DNA-damageinducible transcript 4 (Ddit4) demonstrated 1.9-fold and 2.58fold upregulation in Mir221/222AdipoKO fed with HFHS and STD chow, respectively (Supplementary Table 7). To evaluate miRNA activity of miR-221-3p and miR-222-3p by the binding to 3'-untranslated region (3'-UTR) of Ddit4, we performed luciferase reporter assay in 3T3-L1 cells. We cloned 3'-UTR regions of Ddit4 gene and prepared the wild-type reporter vector, pmirGLO-Ddit4 WT 3'-UTR, and the mutant vector, pmirGLODdit4 MT 3'-UTR, in which mutagenesis was induced on the seed sequence binding site (Figure 3A). The luciferase reporter vectors were co-transfected with Syn-mmu-miR-221-3p or Synmmu-miR-222-3p or negative control siRNA in 3T3-L1 cells. As a result, reporter activity of pmirGLO-Ddit4 WT 3'-UTR were significantly inhibited in the presence of both Syn-mmu-miR221-3p and Syn-mmu-miR-222-3p. In contrast, Syn-mmu-miR221-3p or Syn-mmu-miR-222-3p had almost no effect on the reporter activity of pmirGLO-Ddit4 MT 3'-UTR (Figure 3A). Next, we checked the expression of Ddit4 in the epidydimal adipose tissues of Mir221/222 $2^{\text {flox/y }}$ and Mir221/222AdipoKO fed with HFHS by quantitative PCR and Western blot. Although the mRNA expression of Ddit4 was increased in Mir221/ 222AdipoKO without statistical significance, DDIT4 protein expression was significantly upregulated in the Mir221/ 222AdipoKO compared with Mir221/222 flox/y (Figure 3B), suggesting the possible involvement of translational repression by $\mathrm{miR}-221-3 \mathrm{p}$ and $\mathrm{miR}-222-3 \mathrm{p}$.

\section{Mir221/Mir222 Promotes Adipogenesis by DDIT4-Mediated Inhibition of mTORC1}

DDIT4 is known to activate tuberous sclerosis complex 1 (TSC1)/TSC2 complex by the inhibition of AKT (protein kinase B) and the release of 14-3-3 from TSC2 (12). It further negatively regulates mammalian target of rapamycin complex 1 (mTORC1) (13). Increased activity of the mTORC1 signaling has been associated with obesity (14), and the knockout mice for the mTORC1 downstream ribosomal protein S6 kinase (S6K) are protected against diet-induced-obesity (15). Thus, we further checked the inactivated form of p-TSC2 (Thr-1462) and activated form of p-S6K (16) in the epidydimal adipose tissues of Mir221/222 flox/y and Mir221/222AdipoKO. After overnight fasting, the mice were injected intraperitoneally with insulin and the epidydimal fat tissues were harvested at $7 \mathrm{~min}$ after insulin injection. In Mir221/222AdipoKO mice, both p-AKT and p-TSC2 (Thr-1462) were significantly reduced, and accordingly p-S6K was also significantly reduced (Figures 4A, B). p-mTOR was reduced in Mir221/222AdipoKO mice, however, it did not reach the statistically significant differences (Figures 4A, B).

Since mTOR has been shown to positively regulate adipogenesis and lipogenesis while inhibiting lipolysis, fatty acid oxidation and ketogenesis (17), we performed adipogenesis and glycerol assays in 3T3-L1 adipocytes. We constructed lentiviral vector expressing miRNA inhibitors (pLV-locker 221, pLV-locker 222, pLV-locker control) and miRNA mimics (pLV 221, pLV 222, pLV control). The efficiency for inhibitors and mimics was confirmed in 3T3-L1 cells at 3-7 days after the transduction (Supplementary Figures 3B, C). Lentiviral vectors were transduced to 3T3-L1 cells at 2 days before the induction of differentiation and cultured for 7 days. The pLV-locker 221, pLV-locker 222, and pLV-locker $221 / 222$ did not alter the accumulation of lipid droplets demonstrated by oil red $\mathrm{O}$ staining measured by absorbance at $490 \mathrm{~nm}$ (Figure 5A). Similarly, pLV 221, pLV 222, and pLV221/ 222 only show slight increase in the accumulation of lipid droplets in 3T3-L1 cells without statistical significance (Figure 5B). Western blot analysis demonstrated that the 
A

Mmu-miR-221 5 ' AGCUACAUUGUCUGCUGGGUUUC

Mmu-miR-222 5 ' A GCUACAUCUGGCUACUGGGU I IIIII

pmirGLO-Ddit4 WT 3'-UTR 5 ' TACGATGTATGTGTAGGTCGGTCTTCGG pmirGLO-Ddit4MT 3'-UTR 5 ' TACGTCTATGTGTAGGTCGGTCTTCGG
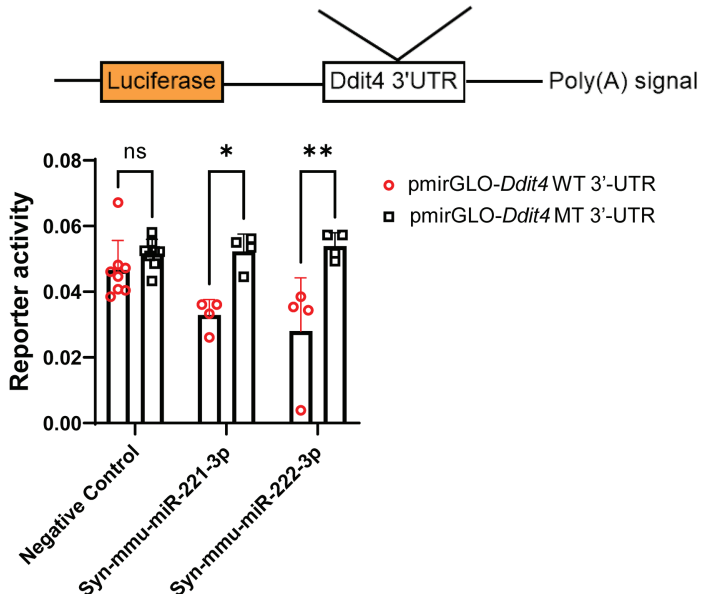

B

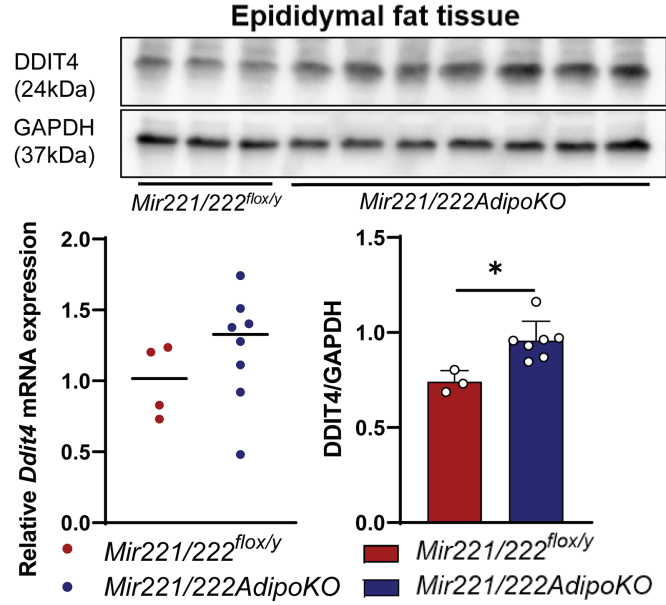

FIGURE 3 | Dual-luciferase reporter assay for Ddit4 3'UTR and expression of Ddit4 in mice. (A) Dual-luciferase reporter assay using pmirGLO-Ddit4 WT 3'UTR, pmirGLO-Ddit4 MT 3'-UTR, and pmirGLO no-insert control plasmids. 3T3-L1 cells were co-transfected with Syn-mmu-miR-221-3p ( $n=4)$, Synmmu-miR-222-3p $(n=4)$, negative control siRNA $(n=8)$. (ns, not significant). (B) Quantitative RT-PCR for Ddit4 in epididymal adipose tissues of Mir221/ $222^{f l o x / y}(n=4)$ and Mir221/222AdipoKO $(n=8)$ mice fed with high fat high sucrose (HFHS). Western blot analyses for DDIT4 and GAPDH (glyceraldehyde3-phosphate dehydrogenase) in epididymal adipose tissues of Mir221/222 floxy $(n=3)$ and Mir221/222AdipoKO ( $n=7)$ mice fed with high fat high sucrose (HFHS). Data shown as mean \pm SD and analyzed by Mann-Whitney's $U$ test $\left({ }^{*} \mathrm{p}<0.05 ;{ }^{* \star} \mathrm{p}<0.01\right)$

expression of $\mathrm{C} / \mathrm{EBP} \alpha$ and $\mathrm{PPAR} \gamma$ were not changed by pLV-locker 221 and pLV-locker 222 (Figure 5C), while they were significantly up-regulated by treatment with pLV 222 (Figure 5D). To investigate lipolysis, lentiviral vectors were transduced to the fully differentiated 3T3-L1 adipocyte and we performed glycerol assay 7 days after lentiviral transduction. pLV-locker 221/222 and pLV 221/222 did not alter the glycerol release (Figure 5E).
A

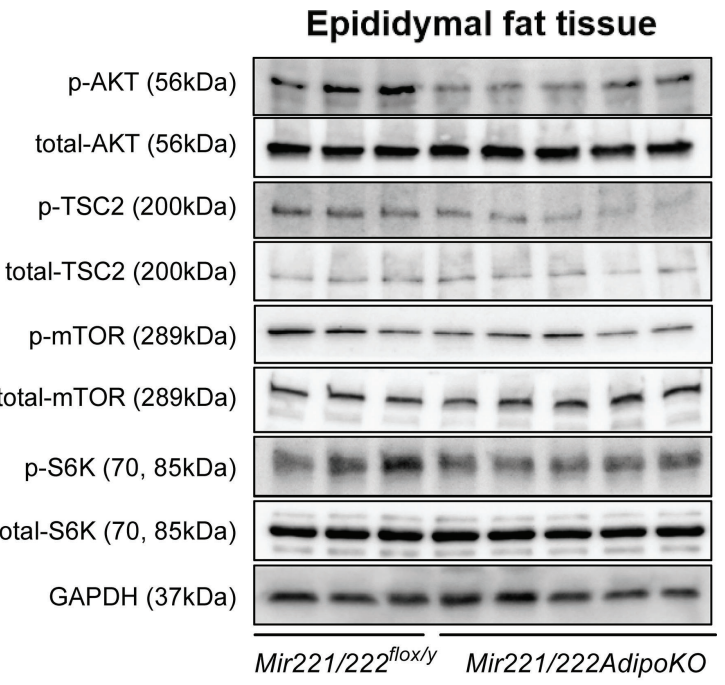

B
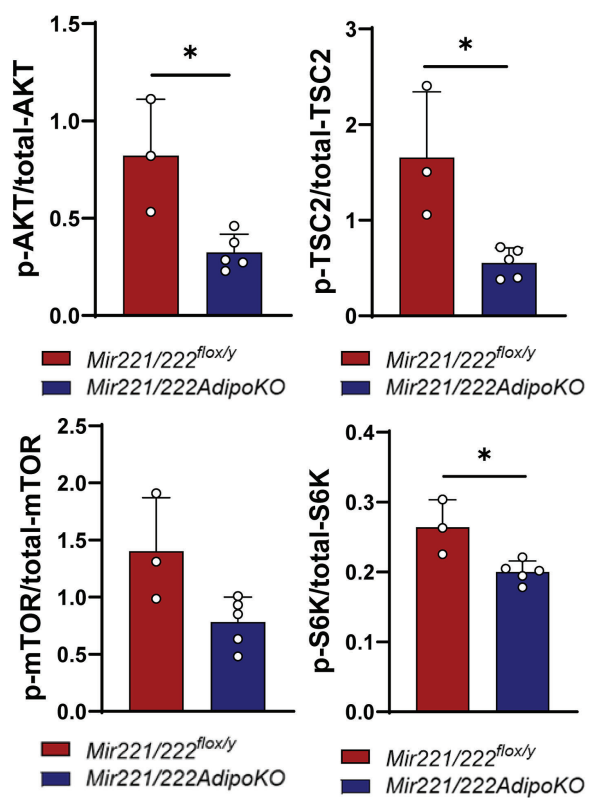

FIGURE 4 | Western blot analyses. (A) Western blot analyses for AKT (protein kinase B), TSC2 (tuberous sclerosis complex 2), mTOR (mammalian target of rapamycin) and S6K (ribosomal protein S6 kinase) in Mir221/222 flox/y $(n=3)$ and in epididymal adipose tissues of Mir221/222AdipoKO $(n=5)$ male mice fed with high fat high sucrose (HFHS). Activated form of $\mathrm{p}-\mathrm{AKT}$ and Inactivated form of p-TSC2 (Thr-1462) and activated form of p-S6K are shown. (B) Densitometric analyses of Western blots. Data shown as mean \pm SD and analyzed by MannWhitney's $U$ test $\left({ }^{*} \mathrm{p}<0.05\right)$.

\section{DISCUSSION}

The adipocyte-specific inhibition of Mir221/Mir222 expression protected the mice fed with HFHS chow from the obesity. We demonstrated that the direct target of miR-221-3p and miR-222$3 p$ was Ddit4 and the inhibition of Mir221/Mir222 expression resulted in upregulation of DDIT4, inactivation of AKT, activation of TSC2 and subsequent inactivation of S6K, the 
A

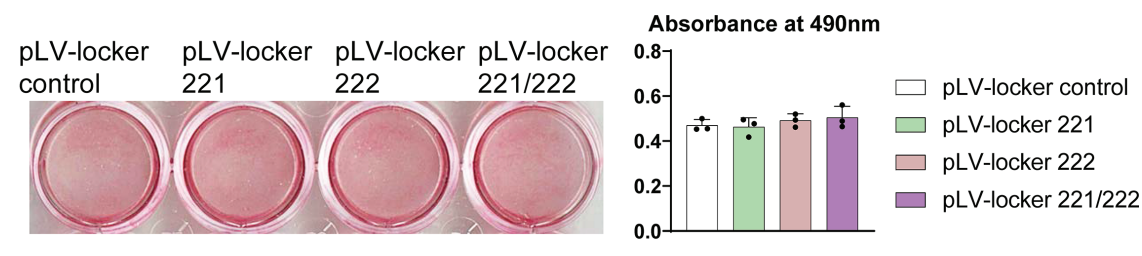

B

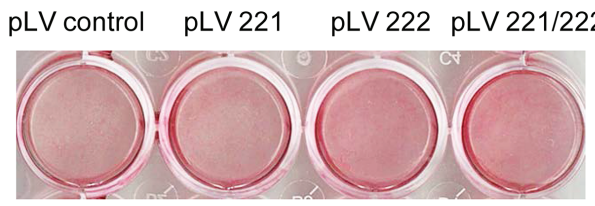

Absorbance at $490 \mathrm{~nm}$

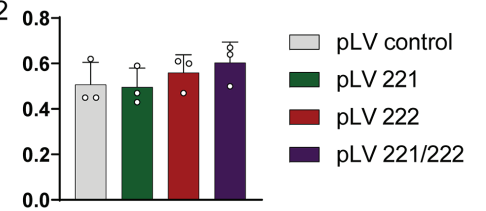

C

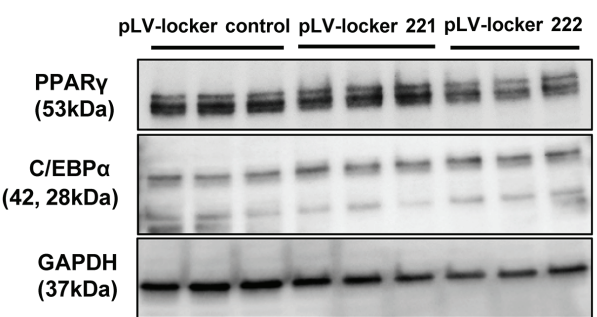

$\square$ pLV-locker control

$\square$ pLV-locker 221

$\square$ pLV-locker 222
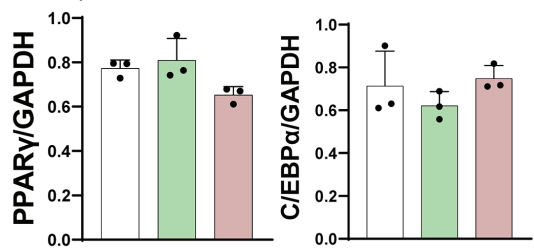

D

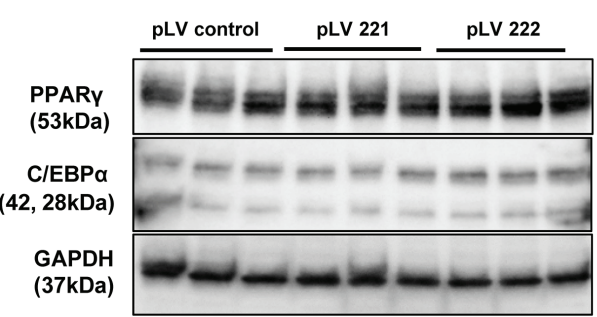

$$
\square \text { pLV control }
$$$$
\text { mLV } 221
$$$$
\text { — pLV } 222
$$
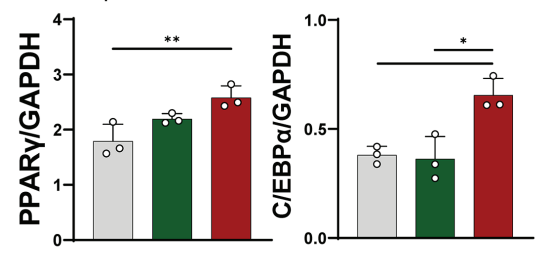

E
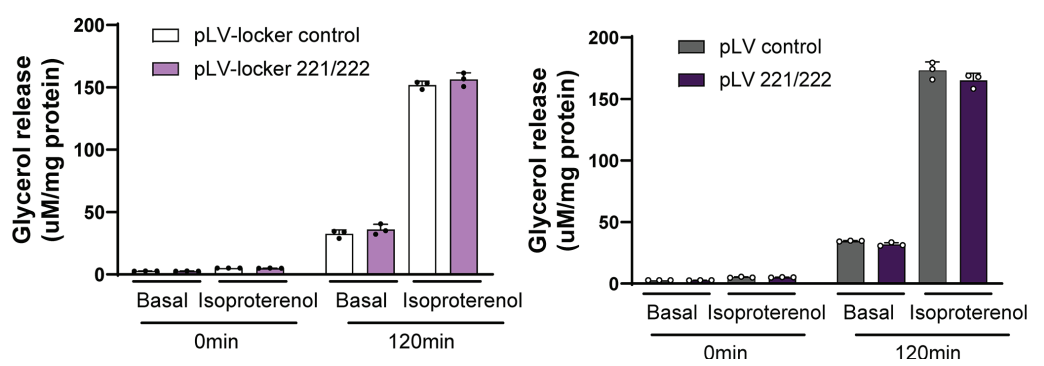

FIGURE 5 | Adipogenesis, lipogenesis and lipolysis in 3T3-L1 cells. Lentiviral vectors were transduced to 3T3-L1 cells at 2 days before the induction of differentiation and cultured for 7 days. (A) Oil-red O staining of differentiated 3T3-L1 cells treated with pLV-locker control, pLV-locker 221, pLV-locker 222, and pLV-locker 221/222. (B) Oil-red O staining of differentiated 3T3-L1 cells treated with pVL control, pLV 221, pLV 222, and pLV221/222. (C) Western blot analysis of PPAR in differentiated 3T3-L1 cells treated with pLV-locker control, pLV-locker 221, pLV-locker 222, and pLV-locker 221/222. (D) Western blot analysis of PPAR in differentiated 3T3-L1 cells treated with pVL control, pLV 221, pLV 222, and pLV221/222. (E) Lipolysis assay in 3T3-L1 cells stimulated with isoproterenol. Differentiated 3T3-L1 cells were treated with pLV-locker 221/222 and pLV 221/222. Data shown as mean \pm SD and analyzed by one-way ANOVA with Tukey test $\left({ }^{*} p<0.05 ;{ }^{* *} p<0.01\right)$.

latter is a major target of mTORC1. The lentivirus-mediated transfer of Mir222 gene promoted the adipogenesis demonstrated by up-regulation of $\mathrm{C} / \mathrm{EBP} \alpha$ and PPAR $\gamma$ in differentiated 3T3-L1 cells. We concluded that the inhibition of miR-221-3p and miR-222-3p and DDIT4-mediated inhibition of mTORC1 signaling is one of the major mechanisms for the protection from diet-induced obesity. The role of Mir221 in adipogenesis has been focused by series of investigation and Ahonen et al. reported miR-221-3p overexpression in SimpsonGolabi-Behmel syndrome (SGBS) preadipocytes inhibited de novo lipogenesis and adipogenesis (18). In our investigation, overexpression by pLV 221 did not alter the lipogenesis and 
adipogenesis in 3T3-L1 cells; however, pLV 222 enhanced the adipogenesis. In bovine adipocyte differentiation, long noncoding RNA (lncRNA), MIR221HG, significantly increased adipocyte differentiation associated with dramatic increment of PPAR $\gamma$ (11). We also cloned mouse Mir221hg with 1,537 bp and identified that $1,054 \mathrm{bp}$ of 5 '-flanking region was deleted in Mir221/222AdipoKO mice. The expression of Mir221hg is barely detected in brown and WATs in wild type mice and we speculated that the influence of deletion of Mir221 hg is minimal in Mir221/222AdipoKO mice. Although the functional studies to elucidate the role of Mir222 in adipogenesis and lipogenesis were not reported in cultured adipocytes in previously published studies, we demonstrated that Mir222 expression was upregulated in WATs of obese mice and in serum of obese patients. The importance of Mir222 in the pathogenesis of obesity is supported by the analysis of adipose tissue-specific Dicer knockout mice, in which gonadal adipose tissue was the main source of serum exosomal miR-222 (19). The exosome-packed Mir222 influenced remote organs, and overexpression of Mir222 inhibits the expression of IRS-1 by directly binding to untranslated regions in the muscle (20) and liver (21). In current investigation, we firstly demonstrated that overexpression of Mir222 promoted the adipogenesis in 3T3L1 cells.

Under the presence of amino acids, mTORC1 is activated by GTP-bound Rheb. In the upstream of the Rheb, AKT inhibits TSC2 and TBC1D7 (TBC1 Domain Family Member 7) complex, which further inhibits the activation of mTORC1 by acting as a GTPase-activating protein. mTORC1 promotes the lipid synthesis and storage and adipogenesis, while it inhibits the lipolysis, $\beta$-oxidation and ketogenesis. SREBP (sterol regulatory element-binding protein) processing and activation is promoted by mTORC1 by the activation of S6K and lipin 1 leading to transcriptional activation of SREPB1, SREBP2, and many other lipogenic genes. mTORC1 also committed mesenchymal stem cells to adipocyte lineage by the activation of $\mathrm{S} 6 \mathrm{~K}$, promotes the initial step of adipocyte differentiation by inhibiting $4 \mathrm{E}-\mathrm{BP} 1 / 2$ (eIF4E-binding protein $1 / 2$ ), and completes the final differentiation by the activation of PPAR $\gamma$ (22). Many researchers screened the agents or intrinsic factors to inhibit mTORC1 signaling and lipogenesis for the treatment of obesity. Lee et al. reported that ezetimibe reduced lipid accumulation by inhibiting mTORC1 signaling, leading to the downregulation of lipogenesis-related genes (23). Shi et al. reported that the inhibition of miR-196b-5p blocked adipogenesis and lipogenesis by directly targeting TSC1 and TGFBR1 (transforming growth factor- $\beta$ receptor 1 ) (24). We demonstrated that the inhibition of miR-221-3p and miR-222$3 p$ and subsequent DDIT4-mediated inhibition of mTORC1 signaling is a therapeutic target for the treatment of obesity.

The activation of mTORC1 links to various pathological processes in adipose tissues such as inflammation, beige adipogenesis and angiogenesis. mTORC1 loss and gain of function studies in macrophages resulted in amelioration and exacerbation of inflammatory response, as well as macrophage polarization to both M1 and M2 profiles (25). miR-221 mediates
M1 macrophage polarization $(26,27)$, while suppression of miR222 alleviate the inflammatory response $(28,29)$. However, we did not observe the apparent changes in gene expression of cytokines such as Illb, Il6, Ifng, and Tnf in adipose tissues in Mir221/222AdipoKO mice (Supplementary Figure 2). The phenotyping of $\mathrm{T}$ cells and macrophages in WATs should be performed in the future investigation. In beige adipogenesis, the adipose-specific deletion of Raptor, a key component of mTORC1, promoted beige adipogenesis by inhibiting prostaglandins (PGs) synthesized by cyclooxygenase-2 (COX-2) (30). Supporting this notion, ketoprofen alleviated diet-induced obesity and promotes the fat browning by the COX-2 and mTORC1-p38 signaling pathway (31). In our investigation, the genes related to beige adipogenesis such as Ppargc1 and Prdm16 tended to be upregulated in WATs without statistical differences. However, in WATs in Mir221/222AdipoKO mice, apparent beige adipogenesis was not observed by histological examinations. In tumor microenvironment and angiogenesis, mTORC1 under a hypoxic condition promotes the translation of hypoxia-inducible factor (HIF) 1-2, which lead to the expression of angiogenic growth factors such as vascular endothelial growth factor (VEGF), TGF- $\alpha$, and platelet-derived growth factor $\beta$ (PDGF- $\beta$ ) (32). Mir221 and Mir222 demonstrated the angiogenic activities in vascular cells (33) and cancers (34), however, we did not observe the angiogenesis activities in WATs in Mir221/222AdipoKO mice.

\section{LIMITATION OF STUDY}

We hypothesized that circulating miR-221-3p and miR-222-3p play important roles in both mouse obese models and the patients with obesity and T2D. However, the circulating levels of miR-221-3p and miR-222-3p were not altered in Mir221/ $222^{f l o x / y}$ and Mir221/222AdipoKO mice fed with STD and HFHS. In addition, the human serum levels of miR-221-3p and miR222-3p showed correlations with HbA1c, however, they did not show significant correlations with body weight and BMI. Although we show the roles of miR-221-3p and miR-222-3p in the adipocytes, we did not clearly demonstrate the role of circulating miR-221-3p and miR-222-3p in the pathogenesis of obesity. Another limitation is that it is still unknown why Mir221/222AdipoKO mice did not show significant improvement of insulin sensitivity although the body weight was significantly reduced compared with Mir221/222 flox/y fed with HFHS. Finally, the micronutrient composition is not matched between the STD and HFHS diets and it may possibly impact the observed outcomes. Ideally, a matched diet should be used for mineral and vitamin mix.

\section{DATA AVAILABILITY STATEMENT}

The datasets presented in this study can be found in online repositories. The names of the repository/repositories and accession number(s) can be found in the article/Supplementary Material. 


\section{ETHICS STATEMENT}

The studies involving human participants were reviewed and approved by Okayama University Graduate School of Medicine, Dentistry and Pharmaceutical Sciences and Okayama University Hospital, Ethics Committee. The patients/participants provided their written informed consent to participate in this study. The animal study was reviewed and approved by the Animal Care and Use Committee of the Department of Animal Resources, Advanced Science Research Center, Okayama University.

\section{AUTHOR CONTRIBUTIONS}

SY, DZ, AK, and JW designed the project and experiments and wrote the manuscript. SY, DZ, AK, NK, RS, and HA performed animal experiments and analyzed and interpreted data. AN and JE performed culture and molecular biology experiments. SY, AK, NK, RS, AN, JE, and JW designed and performed clinical study. All authors contributed to the article and approved the submitted version.

\section{REFERENCES}

1. Kahn CR, Wang G, Lee KY. Altered Adipose Tissue and Adipocyte Function in the Pathogenesis of Metabolic Syndrome. J Clin Invest (2019) 129:39904000. doi: 10.1172/JCI129187

2. Huang Z, Xu A. Adipose Extracellular Vesicles in Intercellular and InterOrgan Crosstalk in Metabolic Health and Diseases. Front Immunol (2021) 12:608680. doi: 10.3389/fimmu.2021.608680

3. Pan Y, Hui X, Hoo RLC, Ye D, Chan CYC, Feng T, et al. Adipocyte-Secreted Exosomal microRNA-34a Inhibits M2 Macrophage Polarization to Promote Obesity-Induced Adipose Inflammation. J Clin Invest (2019) 129:834-49. doi: 10.1172/JCI123069

4. Fu T, Seok S, Choi S, Huang Z, Suino-Powell K, Xu HE, et al. MicroRNA 34a Inhibits Beige and Brown Fat Formation in Obesity in Part by Suppressing Adipocyte Fibroblast Growth Factor 21 Signaling and SIRT1 Function. Mol Cell Biol (2014) 34:4130-42. doi: 10.1128/MCB.00596-14

5. Wang L, Sinnott-Armstrong N, Wagschal A, Wark AR, Camporez JP, Perry RJ, et al. A MicroRNA Linking Human Positive Selection and Metabolic Disorders. Cell (2020) 183:684-701.e14. doi: 10.1016/j.cell.2020. 09.017

6. Qi R, Wang J, Wang Q, Qiu X, Yang F, Liu Z, et al. MicroRNA-425 Controls Lipogenesis and Lipolysis in Adipocytes. Biochim Biophys Acta Mol Cell Biol Lipids (2019) 1864:744-55. doi: 10.1016/j.bbalip.2019.02.007

7. Higuchi C, Nakatsuka A, Eguchi J, Teshigawara S, Kanzaki M, Katayama A, et al. Identification of Circulating miR-101, miR-375 and miR-802 as Biomarkers for Type 2 Diabetes. Metabolism (2015) 64:489-97. doi: 10.1016/ j.metabol.2014.12.003

8. Deiuliis JA. MicroRNAs as Regulators of Metabolic Disease: Pathophysiologic Significance and Emerging Role as Biomarkers and Therapeutics. Int J Obes (Lond) (2016) 40:88-101. doi: 10.1038/ijo.2015.170

9. Villard A, Marchand L, Thivolet C, Rome S. Diagnostic Value of Cell-Free Circulating MicroRNAs for Obesity and Type 2 Diabetes: A Meta-Analysis. J Mol Biomark Diagn (2015) 6(6):251. doi: 10.4172/2155-9929.1000251

10. Nakagawa Y, Ishimura K, Oya S, Kamino M, Fujii Y, Nanba F, et al. Comparison of the Sympathetic Stimulatory Abilities of B-Type Procyanidins Based on Induction of Uncoupling Protein-1 in Brown Adipose Tissue (BAT) and Increased Plasma Catecholamine (CA) in Mice. PloS One (2018) 13:e0201203. doi: 10.1371/journal.pone.0201203

11. Li M, Gao Q, Tian Z, Lu X, Sun Y, Chen Z, et al. MIR221HG Is a Novel Long Noncoding RNA That Inhibits Bovine Adipocyte Differentiation. Genes (Basel) (2019) 11(1):29. doi: 10.3390/genes11010029

\section{FUNDING}

This work was supported by Grant-in-Aid for Young Scientists (19K17984), Grant-in-Aid for Scientific Research (B) (19H03675), Japan Agency for Medical Research and development (AMED, grant no: 17ek0210095h0001, 20ek0109445h0001).

\section{ACKNOWLEDGMENTS}

We acknowledge support from Central Research Laboratory, Okayama University Medical School; usage of Kubota High Speed Refrigerated Centrifuge Model 7780II, ABI PRISM3130, and producing paraffin blocks and sections.

\section{SUPPLEMENTARY MATERIAL}

The Supplementary Material for this article can be found online at: https://www.frontiersin.org/articles/10.3389/fendo.2021.750261/ full\#supplementary-material

12. Britto FA, Dumas K, Giorgetti-Peraldi S, Ollendorff V, Favier FB. Is REDD1 a Metabolic Double Agent? Lessons From Physiology and Pathology. Am J Physiol Cell Physiol (2020) 319:C807-24. doi: 10.1152/ajpcell. 00340.2020

13. Tirado-Hurtado I, Fajardo W, Pinto JA. DNA Damage Inducible Transcript 4 Gene: The Switch of the Metabolism as Potential Target in Cancer. Front Oncol (2018) 8:106. doi: 10.3389/fonc.2018.00106

14. Khamzina L, Veilleux A, Bergeron S, Marette A. Increased Activation of the Mammalian Target of Rapamycin Pathway in Liver and Skeletal Muscle of Obese Rats: Possible Involvement in Obesity-Linked Insulin Resistance. Endocrinol (2005) 146:1473-81. doi: 10.1210/en.2004-0921

15. Yu R, Li Z, Liu S, Huwatibieke B, Li Y, Yin Y, et al. Activation of Mtorc1 Signaling in Gastric X/A-Like Cells Induces Spontaneous Pancreatic Fibrosis and Derangement of Glucose Metabolism by Reducing Ghrelin Production. EBioMed (2018) 36:304-15. doi: 10.1016/j.ebiom.2018.09.027

16. Manning BD, Tee AR, Logsdon MN, Blenis J, Cantley LC. Identification of the Tuberous Sclerosis Complex-2 Tumor Suppressor Gene Product Tuberin as a Target of the Phosphoinositide 3-Kinase/Akt Pathway. Mol Cell (2002) 10:151-62. doi: 10.1016/s1097-2765(02)00568-3

17. Pena-Leon V, Perez-Lois R, Seoane LM. mTOR Pathway is Involved in Energy Homeostasis Regulation as a Part of the Gut-Brain Axis. Int J Mol Sci (2020) 21(16):5715. doi: 10.3390/ijms21165715

18. Ahonen MA, Asghar MY, Parviainen SJ, Liebisch G, Horing M, Leidenius M, et al. Human Adipocyte Differentiation and Composition of Disease-Relevant Lipids Are Regulated by miR-221-3p. Biochim Biophys Acta Mol Cell Biol Lipids (2021) 1866:158841. doi: 10.1016/j.bbalip.2020.158841

19. Li D, Song H, Shuo L, Wang L, Xie P, Li W, et al. Gonadal White Adipose Tissue-Derived Exosomal MiR-222 Promotes Obesity-Associated Insulin Resistance. Aging (Albany NY) (2020) 12:22719-43. doi: 10.18632/aging. 103891

20. de Mendonca M, de Sousa E, da Paixao AO, Araujo Dos Santos B, Roveratti Spagnol A, Murata GM, et al. MicroRNA miR-222 Mediates Pioglitazone Beneficial Effects on Skeletal Muscle of Diet-Induced Obese Mice. Mol Cell Endocrinol (2020) 501:110661. doi: 10.1016/j.mce.2019. 110661

21. Ono K, Igata M, Kondo T, Kitano S, Takaki Y, Hanatani S, et al. Identification of microRNA That Represses IRS-1 Expression in Liver. PloS One (2018) 13: e0191553. doi: 10.1371/journal.pone.0191553

22. Epstein Y, Seidman DS, Moran D, Arnon R, Arad M, Varssano D. HeatExercise Performance of Pyridostigmine-Treated Subjects Wearing Chemical Protective Clothing. Aviat Space Environ Med (1990) 61:310-3. 
23. Lee YS, Park JS, Lee DH, Han J, Bae SH. Ezetimibe Ameliorates Lipid Accumulation During Adipogenesis by Regulating the AMPK-Mtorc1 Pathway. FASEB J (2020) 34:898-911. doi: 10.1096/fj.201901569R

24. Shi Y, Li F, Wang S, Wang C, Xie Y, Zhou J, et al. miR-196b-5p Controls Adipocyte Differentiation and Lipogenesis Through Regulating Mtorc1 and TGF-Beta Signaling. FASEB J (2020) 34:9207-22. doi: 10.1096/ fj.201901562RR

25. Festuccia WT. Regulation of Adipocyte and Macrophage Functions by Mtorc1 and 2 in Metabolic Diseases. Mol Nutr Food Res (2021) 65:e1900768. doi: $10.1002 / \mathrm{mnfr} .201900768$

26. Cai M, Shi Y, Zheng T, Hu S, Du K, Ren A, et al. Mammary Epithelial Cell Derived Exosomal MiR-221 Mediates M1 Macrophage Polarization via SOCS1/STATs to Promote Inflammatory Response. Int Immunopharmacol (2020) 83:106493. doi: 10.1016/j.intimp.2020.106493

27. Quero L, Tiaden AN, Hanser E, Roux J, Laski A, Hall J, et al. miR-221-3p Drives the Shift of M2-Macrophages to a Pro-Inflammatory Function by Suppressing JAK3/STAT3 Activation. Front Immunol (2019) 10:3087. doi: 10.3389/fimmu.2019.03087

28. Zhang H, Luan S, Xiao X, Lin L, Zhao X, Liu X. Silenced microRNA-222 Suppresses Inflammatory Response in Gestational Diabetes Mellitus Mice by Promoting CXCR4. Life Sci (2021) 266:118850. doi: 10.1016/j.lfs.2020. 118850

29. Zhang Y, Yang J, Zhou X, Wang N, Li Z, Zhou Y, et al. Knockdown of miR222 Inhibits Inflammation and the Apoptosis of LPS-Stimulated Human Intervertebral Disc Nucleus Pulposus Cells. Int J Mol Med (2019) 44:1357-65. doi: 10.3892/ijmm.2019.4314

30. Zhang X, Luo Y, Wang C, Ding X, Yang X, Wu D, et al. Adipose Mtorc1 Suppresses Prostaglandin Signaling and Beige Adipogenesis via CRTC2-COX-2 Pathway. Cell Rep (2018) 24:3180-93. doi: 10.1016/j.celrep.2018.08.055

31. Kang NH, Mukherjee S, Jang MH, Pham HG, Choi M, Yun JW. Ketoprofen Alleviates Diet-Induced Obesity and Promotes White Fat Browning in Mice via the Activation of COX-2 Through Mtorc1-P38 Signaling Pathway. Pflugers Arch (2020) 472:583-96. doi: 10.1007/s00424-020-02380-7
32. Conciatori F, Bazzichetto C, Falcone I, Pilotto S, Bria E, Cognetti F, et al. Role of mTOR Signaling in Tumor Microenvironment: An Overview. Int J Mol Sci (2018) 19(8):2453. doi: 10.3390/ijms19082453

33. Poliseno L, Tuccoli A, Mariani L, Evangelista M, Citti L, Woods K, et al. MicroRNAs Modulate the Angiogenic Properties of HUVECs. Blood (2006) 108:3068-71. doi: 10.1182/blood-2006-01-012369

34. Xu CH, Liu Y, Xiao LM, Chen LK, Zheng SY, Zeng EM, et al. Silencing microRNA-221/222 Cluster Suppresses Glioblastoma Angiogenesis by Suppressor of Cytokine Signaling-3-Dependent JAK/STAT Pathway. J Cell Physiol (2019) 234:22272-84. doi: 10.1002/jcp.28794

Conflict of Interest: JW receives speaker honoraria from Astra Zeneca, Daiichi Sankyo, Novartis, Novo Nordisk Pharma, Tanabe Mitsubishi and receives gran support from Astellas, Baxter, Bayer, Chugai, Dainippon Sumitomo, Kyowa Kirin, Novo Nordisk Pharma, Ono, Otsuka, Tanabe Mitsubishi, and Teijin.

The remaining authors declare that the research was conducted in the absence of any commercial or financial relationships that could be construed as a potential conflict of interest.

Publisher's Note: All claims expressed in this article are solely those of the authors and do not necessarily represent those of their affiliated organizations, or those of the publisher, the editors and the reviewers. Any product that may be evaluated in this article, or claim that may be made by its manufacturer, is not guaranteed or endorsed by the publisher.

Copyright @ 2022 Yamaguchi, Zhang, Katayama, Kurooka, Sugawara, Albuayjan, Nakatsuka, Eguchi and Wada. This is an open-access article distributed under the terms of the Creative Commons Attribution License (CC BY). The use, distribution or reproduction in other forums is permitted, provided the original author(s) and the copyright owner(s) are credited and that the original publication in this journal is cited, in accordance with accepted academic practice. No use, distribution or reproduction is permitted which does not comply with these terms. 